\title{
Designing Virtual Instruments with Touch-Enabled Interface
}

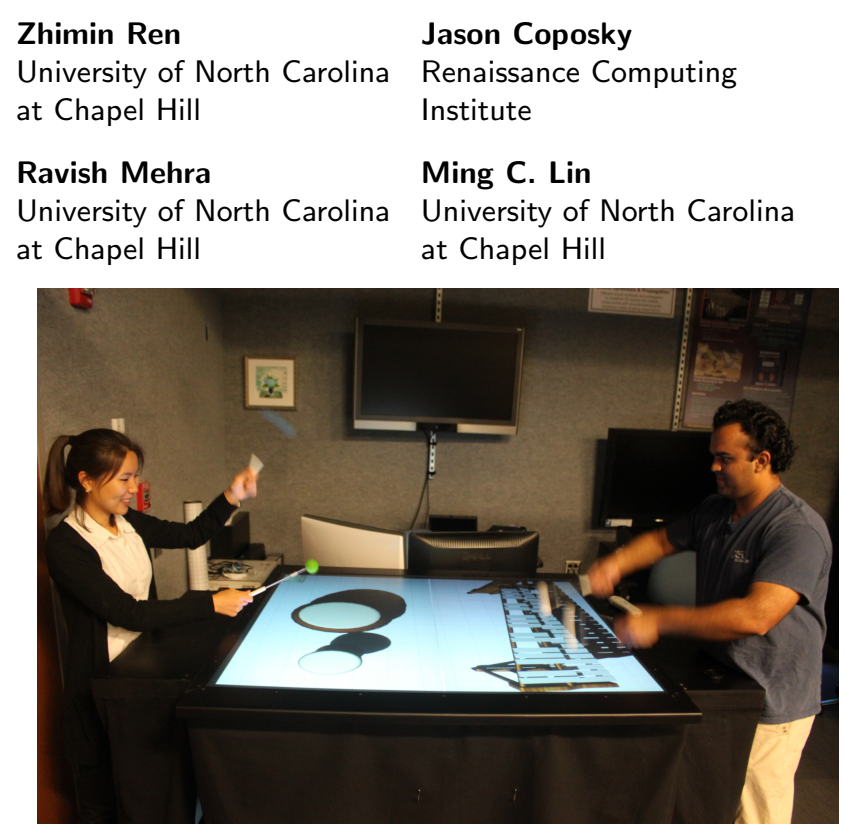

Figure 1: System Setup: Two users collaboratively performing music with our virtual musical instruments.

Copyright is held by the author/owner(s)

CHI'12, May 5-10, 2012, Austin, Texas, USA.

ACM 978-1-4503-1016-1/12/05.

\begin{abstract}
We present and discuss the design of a virtual musical instrument system that can be used by a collaborative group of users to emulate playing percussive music. An optical multi-touch tabletop serves as the input device for multiple users, and an algorithmic pipeline interprets users' interactions with this touch-sensing table and provides control signals to activate the coupled physics-based sound simulation system. The musical tunes can be modulated by our numerical acoustic simulator to create believable acoustic effects generated due to cavity in instruments such as drums. It further allows the users to change the materials, shapes, and sizes of the instruments, thereby offering the capability for both rapid prototyping and active exploration of sound effects by altering various physical parameters. We discuss some of key design principles and what such a system can offer.
\end{abstract}

\section{Keywords}

Multi-touch tabletop; music; multi-modal interaction

\section{ACM Classification Keywords}

H.5.5 [Information interfaces and presentation] Sound and Music Computing;; H.5.2 [Information interfaces and presentation]: User Interfaces. 


\section{Introduction}

In this case study, we present a virtual instrument system using multi-touch interfaces coupled with physically-based sound simulation techniques to offer an alternative paradigm for playing several different virtual instruments simultaneously on the same platform with no overhead. Users' interaction with a multi-touch tabletop naturally represents strike actions, which makes this input device well-suited for percussion instruments. We discuss the key principle considerations in the design of this virtual instrument system that automatically generates realistic audio, directly controlled by users' striking position, playing velocity, striking vigors, and time of impact on the tabletop. In addition, the size of this tabletop system enables multiple users to collaboratively participate in the musical performance simultaneously. Figure 1 shows two users playing virtual percussion instruments on our system. A xylophone of various sounding materials and a set of drums of various sizes and shapes are implemented to demonstrate our results.

This system offers the following unique characteristics over traditional digital instruments:

- A Reconfigurable Platform for Different Instruments and Multiple Players

- Physically-Based Sound Generation

- Direct, Intuitive, Simple Multi-Modal Interfaces

We describe some early user experiences with this system and discuss initial user feedback and suggestions we receive for this virtual instrument system.

\section{Previous Work}

Our paper integrates two large bodies of work. We briefly discuss each area next.
User Interface for Virtual Musical Instruments Researchers have shown that the integration of multi-modal information is essential for musical performance. Chuchacz et al. [2] designed an electronic percussion synthesizer, and Hsiao and Paradiso [4] developed a musical controller using wireless magnetic tags. Both tools are targeted towards amateur users and both include new hardware for a performer to use as musical interface. These two papers explored the possibility of representing musical gestures in virtual reality in a novel way. Miranda and Wanderley [5] assess digital musical instruments with the definition that it is an instrument with a separate control surface from sound production surface. Recently, much work has been done on creating new tools for representing musical gestures in virtual reality to allow control of a music instrument. Some work has been done on analyzing available user interfaces and evaluating the gestural controllers. Poepel and Overhold [6] showed that it is important that gestures be intuitive, powerful, and perceptive. Our work mimics the pre-digital musical instrument era by allowing users to use traditional musical gestures on our virtual instruments as if the control surface is the real sound production surface. This approach enables the user to use natural, intuitive gestures, instead of having to learn new gestures, while providing the advantages of a virtual instrument.

\section{Sound Simulation for Virtual Instruments}

For real-time, physically-based sound synthesis, Van den Doel and Pai [9] introduced a general framework using resonance modes, i.e. modal synthesis $([1,8])$, to generate sound dependent on the materials, shapes, and strike positions of the simulated sounding objects. There is also abundant research on sound synthesis for digital instruments. Most methods concentrate on simulating digital instruments through various types of digital signal 

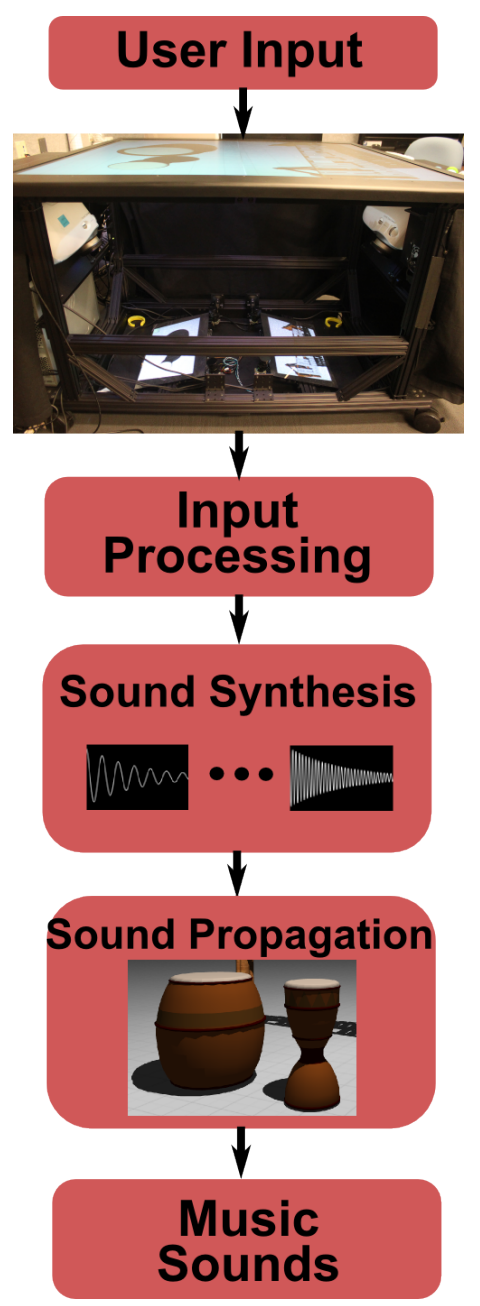

Figure 2: Pipeline: User input is detected by multi-touch table and processed to generate excitation to sound synthesis and propagation engines accordingly. Realistic music sounds corresponding to user inputs are automatically simulated. processing, modal synthesis applied to simple shapes (e.g. strings, tubes, membranes, and more), etc. Cook [3] provided an excellent review on this topic.

\section{System Overview}

Figure 2 outlines the major components in our system.

\section{Multi-Touch Sensing}

Our application is developed on top a custom-built multi-touch table by Renaissance Computing Institute. The table allows us to track multiple (up to 20 or so) touches on the table, by both objects and fingers. The table uses diffuse side illumination for touch detection. It has a 62" diagonal work surface and is 40" tall (see picture in Fig. 2) and comfortably allows 4-6 people to work at the table, as well as allowing a dozen people to stand around it. This optical multi-touch table only detects user inputs as occluded area on the tabletop.

Contact pressure or the velocity at which users are hitting cannot be detected. However, these parameters are critical to music performance modeling which requires information like how rigorous the user is hitting the instrument.

\section{Input Processing and Interpretation}

We propose to use deformable bodies to convey pressure and hitting velocity through optical multi-touch surfaces. Specifically we use the hitting velocity as the indicator of how hard users are striking the instruments. We choose velocity over pressure, because pressure at one instance does not necessarily reflect how hard users are hitting the surface, e.g. users can be statically pressing against the surface, and this should not excite the virtual sounding objects. Registering velocity through an optical device is more challenging and less straightforward than pressure. We create buffers to record a sequence of occlusion information of the deformable strikers registered by the multi-touch system. The record information including the center position, the occluded area, and the time stamp of an occlusion event. One buffer is created for all occlusion data centered within a small position range, as they are considered as one strike. By looking through the occlusion variation over time in one particular buffer, we can derive the average contact velocity representing how fast the soft body is hitting the hard surface in one touch. With this approach, we can easily track many simultaneous touches from multiple fingers and users to generate corresponding excitation to our sound simulation engine.

\section{Sound Simulation}

Modal analysis and modal synthesis techniques are adopted for physically-based approximating vibrations of sounding components in the simulated instruments. This enables creating virtual instruments of various materials and shapes in preprocessing and also generating richly varying and interaction-dependent audio at run-time. Precomputed wave simulation methods proposed by Raghuvanshi et al. [7] is used in our system to simulate acoustic effects in instrument cavity.

\section{Discussion and Conclusion}

One of the key considerations in designing this virtual instrument system is the types of instruments that can best take advantages of the interaction offered by a multi-touch tabletop. We focused the implementation of our system around percussion musical instruments, as the mechanism and skills to play such instruments are similar to the interaction with a multi-touch system. This is critical in providing users similar experiences as the real instruments, which minimizes the learning curve for using the system.

We invited people of different age groups and various 
music playing backgrounds, from novice music players to professional musicians, to play our virtual instrument system. All users were able to start generating music right away by striking the simulated xylophone and drum set with mallets or fingers without any significant familiarization with the setup. When up to six users are standing around the table and interacting with the virtual instruments simultaneously, the system can still successfully handle all user inputs and generate corresponding music sounds in real-time. In cases where experienced music players use the system, they can collaborate and create music pieces easily with the multi-instrument setup as they do with real percussion instruments. Some example performances can be found on this web page.

In conclusion, we present a virtual instrument system that enables multiple users to collaboratively perform music simultaneously. It uses an efficient and responsive approach that interprets the user input from an optical multi-touch interface and generates excitation information for real-time sound simulation that creates realistic sounds depending on striking position, impact vigor, and the shape, material, and cavity of instruments. While our current hardware setup suits collaborative purposes in scenarios like museums and schools, these design principles can be easily adopted to run on other input devices, such as multiple tablet PCs, iPads, or other multi-touch displays. Based on early user feedback, this multi-modal system is intuitive, easy-to-use, and fun to play with for novice users and experienced musicians alike. In future, we can also offer users to change instruments' material and shape to create customized virtual percussion instruments. Further investigation can be done towards building systems that handle interactions for other types of musical instruments, e.g. string and reeds.

\section{References}

[1] J.-M. Adrien. Representations of musical signals. chapter The missing link: modal synthesis, pages 269-298. MIT Press, Cambridge, MA, USA, 1991.

[2] K. Chuchacz, S. O'Modhrain, and R. Woods. Physical models and musical controllers: designing a novel electronic percussion instrument. In NIME '07: Proceedings of the 7th international conference on New interfaces for musical expression, pages 37-40, New York, NY, USA, 2007. ACM.

[3] P. Cook. Real sound synthesis for interactive applications. AK Peters, Ltd., 2002.

[4] K. Hsiao and J. Paradiso. A new continuous multimodal musical controller using wireless magnetic tags. In Proc. of the 1999 International Computer Music Conference, pages 24-27. Citeseer, 1999.

[5] E. Miranda and M. Wanderley. New digital musical instruments: control and interaction beyond the keyboard. AR Editions, Inc., 2006.

[6] C. Poepel and D. Overholt. Recent developments in violin-related digital musical instruments: where are we and where are we going? In NIME '06: Proceedings of the 2006 conference on New interfaces for musical expression, pages 390-395, Paris, France, France, 2006. IRCAM - Centre Pompidou.

[7] N. Raghuvanshi, J. Snyder, R. Mehra, M. Lin, and N. Govindaraju. Precomputed wave simulation for real-time sound propagation of dynamic sources in complex scenes. ACM Trans. Graph., 29:68:1-68:11, July 2010

[8] A. Shabana. Vibration of discrete and continuous systems. Springer Verlag, 1997.

[9] K. Van Den Doel and D. Pai. The sounds of physical shapes. PRESENCE-CAMBRIDGE MASSACHUSETTS-, 7:382-395, 1998. 